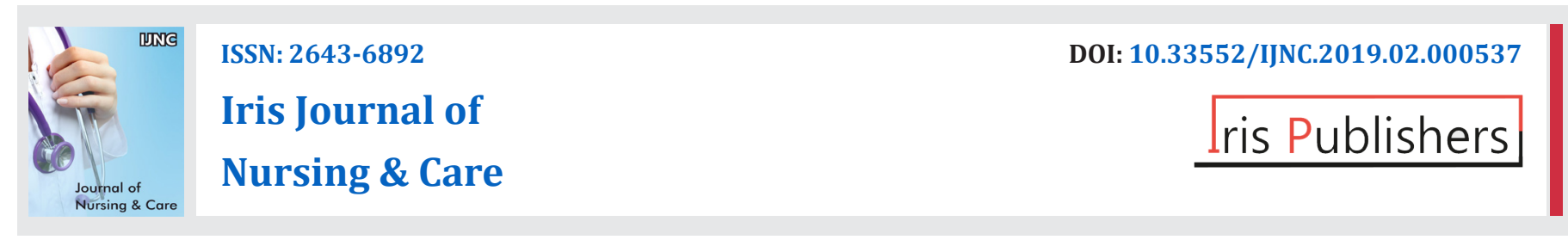

Mini Review

Copyright (C) All rights are reserved by Larider Ruffin

\title{
The Roles of the Nurse During the Vaping Crisis
}

\author{
Larider Ruffin* \\ Assistant Professor of Nursing, Wilmington University, Delaware, USA
}

*Corresponding author: Larider Ruffin, Assistant Professor of Nursing,

Wilmington University, Delaware, USA.

Received Date: November 19, 2019

Published Date: December 17, 2019

\section{Introduction}

Electronic cigarettes (E-cigarettes) are battery-operated devices that transport a nicotine-containing aerosol or vapor by heating the liquid. The liquid usually contains nicotine, propylene glycol or glycerol, chemicals, and a flavoring agent. Additionally, e-cigarettes are used to vape illicit substances such as cannabis. When the chemicals are heated, they convert to toxic aldehydes that cause lung disorders, inflammation, and upper airway irritation. Some of the flavorings for e-cigarettes contain chemicals that can cause inflammatory obstruction of the bronchioles. This is called bronchiolitis obliterans (popcorn lungs). Bronchiolitis obliterans is an injury to the small airways. The signs and symptoms of bronchiolitis obliterans are cough, dyspnea, wheezing, and fatigue. The symptoms are usually slow and progressive (Figure 1) [1-3].

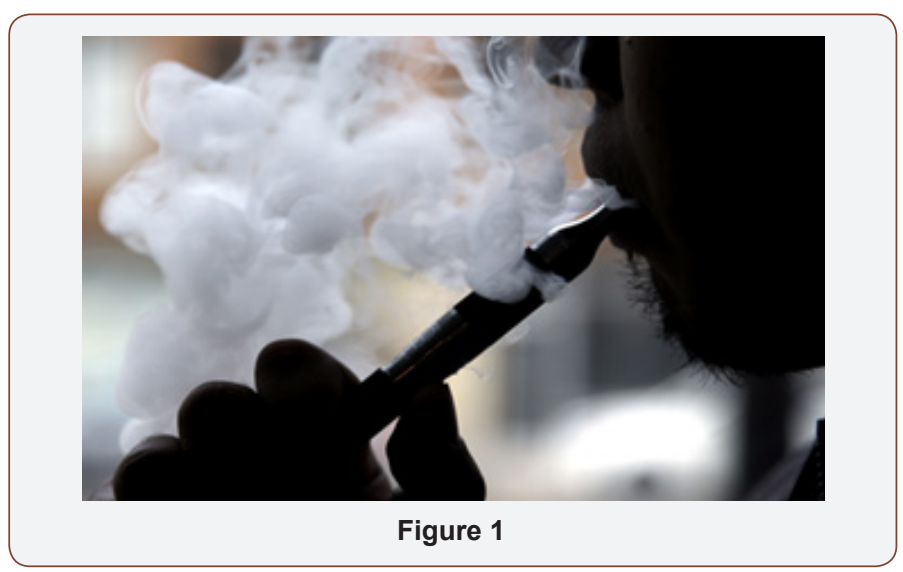

\section{Trends in Vaping}

E-cigarette use increased in high school students in the United States (U.S) from $11.7 \%$ in 2017 to $20.8 \%$ in 2018 . E-cigarette use increased in middle school students in the U.S from $3.3 \%$ in 2017 to $4.9 \%$ in 2018. Approximately 3.62 million middle and high school students were current users of e-cigarettes in 2018 (Food and Drug Administration [FDA], 2019) [4]. A large national survey administered annually to students respectively in $8^{\text {th }}$ grade, $10^{\text {th }}$ grade, and $12^{\text {th }}$ grade found vaping prevalence more than doubled in each of the three grades from 2017 to 2019 [5]. With e-cigarettes marketing campaigns capturing the attention of youth, vaping among middle school and high school students has exceeded cigarette use. Current advertising and health debates about e-cigarette use do not include the negative health effects of nicotine addiction and the vulnerability of young people to nicotine because their brains are in a critical time of development [1].

\section{Vaping Flavored e-cigarettes}

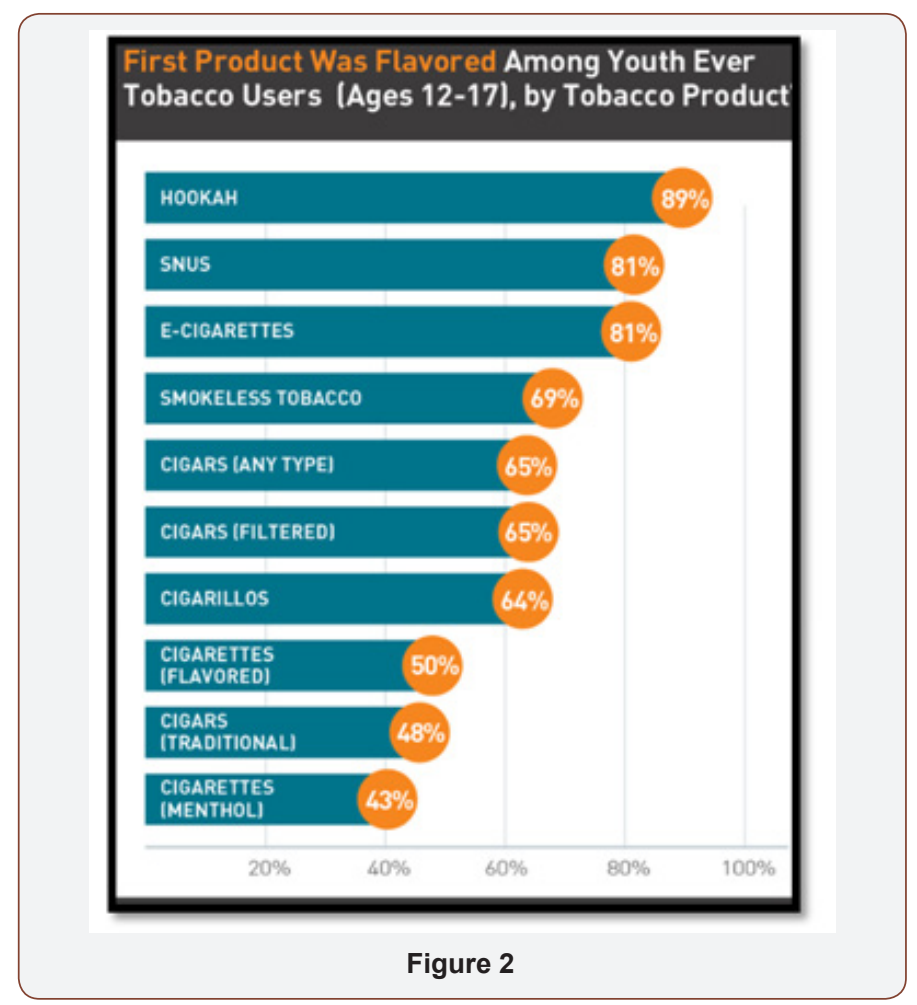

Many tobacco companies claim the flavoring in electronic cigarettes is in food products which consumer ingests daily. While this claim may be truth, food products in the pulmonary system is 
associated in health hazards. Diacetyl and acetyl propionyl found in flavoring may be safe to eat, data suggest, however, that they are not safe when vaporized and breathed (National Institute for Occupational Safety and Health, CDC) [6]. Unfortunately, the youth vaping crisis is directly related to the appeal of flavored e-cigarettes (CDC, 2019) [7]. A recent survey attests 97\% of youth e-cigarette users report using a flavored product in the past month while $70 \%$ cite flavors as the reason for their use. Many teens and pre-teens vape due to flavoring. Unfortunately, there are over 8,000 different types of flavors on the market. The youth used different types of Electronic Nicotine Delivery Systems (ENDS) which include electronic cigarettes to inhale their flavored nicotine (Figure 2).

\section{The Outbreak}

As of November $1^{\text {st }}, 2019$, the CDC has investigated samples of bronchoalveolar lavage (BAL) fluid from 29 injured patients represented from 10 States. Vitamin E acetate has been identified in $100 \%$ of the sample, THC in $82 \%$, while nicotine was identified in $62 \%$ of the sample (CDC, 2019) [7]. The investigation continues and spans across nearly all states, involves over 2,000 injured patients. Forty-four deaths have been reported in 24 states and the District of Columbia. The age of deceased patients ranged from 17 to 75 year of age with $40 \%$ of cases between the ages of 18 and 24, 25\% between 25 and 34 .

\section{The Roles of the Nurse}

The roles of the nurse go far beyond caring for the sick. The nurse should always be willing and ready to apply the systematic guide of the nursing process while advocating for the vulnerable population. The nurse, as the most trusted professional across healthcare, should advocate at the Federal, State, and local levels by partnering with Legislators and advocate for change. We need to encourage legislation to prevent the sales, marketing, and use of e-cigarettes.

During the vaping crisis, if a patient has been vaping and experienced respiratory issues such as a cough, shortness of breath, wheezing, or chest pain, they should go to the Emergency Department (ED) immediately. Patients that vape should tell their primary care provider (PCP) of any of the symptoms immediately for further direction. The role of nurses during the vaping crisis is to be knowledgeable about vaping, advocate for patients, follow institutional protocol, and if bronchiolitis obliterans known as popcorn lung is suspected in the community, patients should be referred to the ED for prompt evaluation. As the most trusted professionals, nurses should support patients to stop smoking and vaping [3].

Nurses should know that the nicotine in e-cigarettes varies from 0 to $36 \mathrm{mg} / \mathrm{ml}$. Even the so-called nicotine-free products have been shown to contain nicotine, and heating e-liquid which elevates temperatures increases nicotine release with negative effects. When nurses are evaluating patients with respiratory issues, they should ask patients if they have used e-cigarette products or vaped in the last 3 months. If the patients say "Yes," nurses should ask about the substances used (homemade liquid, re-used old cartridges, commercially purchased liquids, etc.), the brand name, purchased location, whether e-cigarettes were shared with others. The nurse should act accordingly and report to the Department of Health.

\section{Acknowledgement}

None.

\section{Conflict of Interest}

No Conflict of interest.

\section{References}

1. Duderstadt K (2015) E-Cigarettes: Youth and Trends in Vaping. J Pediatr Health Care 29(6): 555-557.

2. Gonzalvo J, Constantine B, Shrock N, Vincent A (2016) Electronic Nicotine Delivery Systems and a Suggested Approach to Vaping Cessation. AADE in Practice 4(6): 38-42.

3. Schnur M (2019) Vaping Epidemic: A Public Health Crisis.

4. US Food \& Drug Administration (2019) Vaporizers, E-Cigarettes, and other Electronic Nicotine Delivery Systems (ENDS).

5. Miech R, Johnston L, O Malley PM, Bachman JG, Patrick ME (2019) Trends in Adolescent Vaping, 2017-2019. N Engl J Med 381(15): 1490-1491.

6. National Institute of Health office of dietary supplements (2019) Vitamin E Fact Sheet for Health Professionals.

7. CDC (2019) Outbreak of Lung Injury Associated with the Use of E-Cigarette, or Vaping, Products. 